\title{
La consolidación de prácticas de mercado en la educación chilena a partir de una política educativa des-mercantilizadora.
}

\author{
Mateluna Estay, Hernán ${ }^{1}$ \\ Universidad Autónoma de Barcelona, Barcelona, España \\ hernanmateluna@gmail.com
}

\section{Resumen}

El sistema educativo chileno se ha establecido como uno de los más privatizados en el mundo. Sin embargo, a causa de las protestas de los movimientos estudiantiles de los años 2006 y 2011 por acabar con las prácticas de mercado en educación, el gobierno en el año 2014 promete una reforma educacional para cumplir dicha demanda. La primera ley de esta reforma es la denominada «ley de inclusión escolar», la que se constituye en el punto de partida en el proceso de des-mercantilización del sistema educativo chileno. Este trabajo analiza el proceso de construcción y aprobación de una política «des-mercantilizadora» en uno de los sistemas educativos más privatizados del mundo. Le investigación se fundamenta en el enfoque analítico de la Economía Política Cultural aplicada a la adopción de políticas educativas. A nivel metodológico el trabajo examina documentos oficiales relacionados con la elaboración de la ley, así como también los fundamentos teóricos que utilizó el gobierno para justificarla. El trabajo examina la trayectoria de la ley, las disputas por lograr su aprobación y la oportunidad de consensuar entre la derecha y la izquierda la consolidación de prácticas de mercado en el sistema educativo.

\section{Abstract}

The Chilean educational system has been set as one of the most privatized in the world. However, due to the student movements from to 2006 to 2011 where they requested to end the practices of market in education, the Government in 2014 promised an educational reform to meet that demand. The first law of this reform is the named «school inclusion law», that is the starting point in the process of diminishing the market dynamics of the Chilean educational system. This paper analyses the process of construction and approval of a «de-commoditizing» policy in one of the most privatized educational systems in the world. Theoretically, the paper is based on the analytical approach of the Cultural Political Economy applied to adopting educational policies. Methodologically it examines official documents relating to the drafting of the bill, as well as the theoretical foundations that the Government used to justify it. The paper examines the trajectory of the law, the disputes to obtain its approval and the opportunity to obtain consensus in the consolidation of market practices in the education system between the right and left party.

Palabras clave: Chile, desmercantilización, educación, mercado escolar, ley de inclusión.

Keywords: Chile, decommodification, education, school market, school inclusion law.

\section{INTRODUCCIÓN}

Durante la dictadura chilena, en la década de los 80 , se inicia una transformación del sistema educativo basándose en las concepciones neoliberales de Milton Friedman (Vergara, 2012). Esta transformación, reduce la función del Estado a un rol subsidiario (Ruiz, 2010) porque es el mercado el que pasa a ser el principio regulador del sistema educativo (Ruiz, 2012). Así se configura un sistema descentralizado de educación (Rodríguez, 2005) que se fundamenta en la libertad de elección que tienen las familias para elegir la escuela donde enviarán a sus hijos a través del sistema de cheque escolar "voucher» (Friedman, 1955) y un sistema de financiamiento por parte del Estado de subvenciones a la demanda en función al servicio prestado para asegurar la competencia entre las diversas escuelas (Jofré, 1988). 
Este sistema de financiamiento por parte del Estado era el mismo para las escuelas públicas y particulares subvencionadas ${ }^{2}$. Esta igualdad de trato termina otorgando grandes ventajas al sector particular subvencionado (Falabella, 2015), tales como poder lucrar con fondos públicos, cobrar una mensualidad a las familias y seleccionar a sus estudiantes. En este contexto las escuelas particulares subvencionadas aumentaron su matrícula en desmedro de la educación pública debido a la fuga de los estudiantes de medianos ingresos (Canales, Bellei, \& Orellana, 2016; Hsieh \& Urquiola, 2006; Valenzuela, Villalobos, \& Gomez, 2013), lo que ha repercutido en la segregación del sistema educacional chileno hasta llegar a ser uno de los más segregados del mundo (OECD, 2013).

Este contexto fue puesto en entredicho por los movimientos estudiantiles de los años 2006 y 2011. Como respuesta, se instala la necesidad de modificar el sistema educativo mediante una reforma educacional, la cual de acuerdo a la cuenta pública anual realizada por el gobierno en el año 2015, busca el fortalecimiento de la educación pública y terminar con la lógica de mercado en el sistema educativo chileno (Ministerio de Educación, 2015).

Esta reforma educacional está pensada por etapas o leyes independientes que en su conjunto conforman la reforma. La primera ley corresponde a la $N^{\circ} 20.845$ de inclusión escolar, la cual modifica diversos cuerpos legales y establece 38 artículos transitorios. Esta ley es el punto de partida dentro del proceso de «desmercantilización» del sistema educativo chileno, la cual establece la eliminación de tres prácticas que considera de mercado de la educación que recibe subvención estatal: lucro, selección escolar y financiamiento compartido.

\section{APROXIMACIÓN METODOLÓGICA}

Esta investigación analiza la trayectoria desde la elaboración hasta la promulgación de la ley de inclusión escolar, utilizando el modelo establecido por Jessop (2010) a través de herramientas conceptuales de la economía política cultural respecto a la interacción que existe entre los factores materiales y semióticos en los mecanismos evolutivos de selección, retención y variación que permiten explicar las transformaciones institucionales. Estos mecanismos de análisis han sido desarrollados en diferentes contextos para analizar la adopción de las políticas educativas. y que posteriormente es desarrollado por Verger (2016) para el análisis de políticas educativas (Verger, Zancajo, \& Fontdevila, 2016). Para la investigación sobre la trayectoria de la ley de inclusión escolar se analizaron discursivamente documentos relacionados con el proceso de elaboración de la misma teniendo como patrones preestablecidos los conceptos de lucro, selección y financiamiento compartido o copago.

\section{VARIACIÓN. EL SENTIDO COMÚN DEL NEOLIBERALISMO EN ENTREDICHO}

El primer momento de la adopción de políticas corresponde a la variación, en la cual como resultado a situaciones criticas e insatisfactorias en el sistema educativo, se revisan las prácticas políticas dominantes y emergen nuevos discursos y propuestas para responder las demandas. En la etapa de variación de la actual reforma educativa chilena, se reconocen dos momentos claves de crisis en el sistema educativo que propiciaron su emergencia: los movimientos estudiantiles de los años 2006 y 2011. Ambos movimientos lograron influir en las políticas públicas propiciando la reforma educacional que se propone desmercantilizar la educación y proponerla como un derecho social (Bellei, 2016; Picazo \& Pierre, 2016).

En un primer momento, el movimiento de escolares del 2006 logra una gran amplitud y cuentan con el apoyo masivo de la ciudadanía, sin embargo los cambios que consiguen no alteran la lógica neoliberal del modelo educativo (Garreton et al., 2011) por el contrario, la conducción que dio la clase política al conflicto finalmente permitió la intensificación de otro tipo de políticas de mercado como la de rendición de cuentas (Parcerisa \& Fallabella, 2017).

\footnotetext{
${ }^{1}$ Este trabajo se ha realizado en el marco del Programa de Doctorado en Educación de la UAB y ha sido financiado por CONICYT BECAS CHILE 72170165.

${ }^{2}$ Escuelas administradas de forma privada que reciben subvención estatal.
} 
Ante la insatisfacción por los cambios producidos y con la maduración del proceso anterior (Bellei, Cabalin, \& Orellana, 2014), surge en el año 2011 un movimiento de universitarios que van a incorporar las propuestas de los escolares sobre la defensa de la educación pública y el fin al lucro en el sistema educativo (García-Huidobro, 2011). Este nuevo movimiento no se va a conformar con la «política de los consensos» (Moulian, 1997) sino que ahora la demanda se articula exigiendo que el modelo educativo de mercado sufra un «cambio de paradigma» (Boric, 2012).

En el corto y largo plazo, estos movimientos lograron impactar y modificar la política educativa chilena (Cabalin \& Bellei, 2013), no solo como movimiento, sino posteriormente como parlamentarios, ya que cuatro de esos lideres estudiantiles llegarían en el año 2014 al parlamento y participaran activamente en la promulgación de las leyes de la reforma. Esta nueva generación de líderes, que nacen o crecen ya en democracia, no se sentían coartados por esta idea de «democracia limitada» (Falabella, 2015, p. 706) que condicionó los cambios en el retorno a la democracia. Para ellos la hegemonía de las ideas neoliberales en educación no era de sentido común (Cabalin, 2012), por lo que exigieron acabar con el sistema de mercado en educación.

\section{SELECCIÓN. LA EDUCACIÓN COMO DERECHO SOCIAL}

La segunda etapa en el proceso de adopción de políticas es la selección. Esta es la fase en que ya habiendo interpretado la crisis, los policy makers elijen determinadas soluciones políticas que se consideran mas adecuadas para solucionarla y se descartan otras. Debido a que el movimiento del 2011 no logra que el gobierno de centro-derecha haga cambios estructurales en educación, esto significó una oportunidad para que la centro-izquierda rearticulase su discurso de cara a las elecciones del 2013, tomando las demandas del movimiento (Segovia \& Gamboa, 2012) y posteriormente incluyéndolas en el programa de gobierno de la por ese entonces candidata Bachelet (Bellei, 2016). En su programa Bachelet se comprometía a realizar tres reformas estructurales: tributaria, de educación y constitucional. La reforma tributaria es la que permitiría financiar a la educativa, y así poder cumplir con lo ambicioso de está última, ya que busca «un cambio de paradigma» que «implica pasar de la educación como un bien que es posible transar en el mercado... a un sistema educacional coordinado que ofrece a las niñas, niños y jóvenes de Chile un derecho social» (Bachelet, 2013, p.17).

Asimismo, en esta etapa juegan un papel determinante «una gama de factores ideacionales» (Verger, 2016, p. 72), tales como ideologías, marcos o paradigmas políticos. En el caso de la reforma educativa y de la ley de inclusión escolar en Chile, ese factor ideacional fue la idea del derecho social. Esta idea atribuible a T.H. Marshall (1950) nos dice que hay ciertos aspectos mínimos de bienestar esenciales para todas las personas, tales como la educación, salud o vivienda, que no dependen de su renta, sino del estatus de ciudadano. Fernando Atria, uno de los autores intelectuales de la reforma, encuentra en los derechos sociales la forma de superar la desigualdad producida por la educación entendida como bien de mercado, ya que la educación como derecho social significa que todos por ser ciudadanos tienen el mismo derecho a ella; en cambio cuando la educación es un bien de mercado, esta se distribuye según la capacidad de pago de las familias (Atria, 2014). Este nuevo contexto que piensa la educación como un derecho social, corresponde para Atria a un nuevo de paradigma en educación que reemplaza al neoliberal, sin estatizar la educación ni suprimir al sector privado, solo que les exige adecuarse al «régimen de lo público» eliminando el lucro, la selección escolar y el financiamiento compartido (Atria et al, 2013; Atria, 2014).

El proceso de adopción de esta política dejó de manifiesto la alta privatización que alcanzó el sistema educativo chileno, puesto que Chile se establecía como una «sociedad de referencia» (Schriewer, 1993) al ser el único país en el mundo donde escuelas que reciben fondos públicos lucran, seleccionan y cobran a los padres (Ignacio Walker, Historia de la Ley $N^{\circ} 20.845$, p.753) En efecto, en esta etapa en donde las influencias internacionales son más visibles (Verger, 2016), ocurría que no había ningún país que sirviera como ejemplo para justificar el mantenimiento del modelo educativo.

\section{RETENCIÓN: CAMBIO DE PARADIGMA EN LA MEDIDA DE LO POSIBLE}

La última etapa en la adopción de políticas corresponde a la retención. Esta es la fase de negociación para que las políticas educativas sean institucionalizadas en el marco regulatorio. Para Verger (2016) esta es la etapa potencialmente más crítica, ya que surgen con más fuerza los movimientos de oposición que pueden «desplazar» (p.74) los planes del gobierno. 
El gobierno contaba con la mayoría para lograr la aprobación de la ley, pero debía alinear a su propia coalición para lograr sacar adelante el proyecto, ya que estaba formada en un extremo por el Partido Comunista y en el otro por la Democracia Cristiana, cercana al centro. En el caso chileno, al igual que sucede en otros contextos, las coaliciones son tan diversas ideológicamente, que resulta muy difícil llegar a acuerdos para tomar medidas drásticas como la privatización de la educación. En este caso esa diversidad suponía una dificultad para llegar a acuerdos para desprivatizar la educación, que ha sido un emblema del paradigma neoliberal experimental chileno, aunque son lógicas expandidas a nivel global (Luengo, Olmedo, Santa Cruz \& Saura, 2012). Esto sumado a la falta de acuerdos con la oposición, llevo a que la ley sufriera modificaciones que incluyeron aproximadamente seiscientas indicaciones (Bachelet, 2015). Dentro de esos cambios, ya sea al proyecto de ley o a las mismas indicaciones sugeridas en los trámites parlamentarios, la ley sufrirá un "desplazamiento» respecto a su espíritu desmercantilizador que detallamos con los conceptos de fin al lucro, fin a la selección y el fin al financiamiento compartido.

La eliminación del lucro en la educación subvencionada quedó básicamente sujeto a la imposibilidad para que los dueños de escuelas puedan extraer utilidades con la subvención que entrega el Estado, dejando afuera otras formas de lucrar con esos recursos como la contratación de servicios externos como puede ocurrir por ejemplo con las Agencias de Asistencia Técnico.

Educativas (ATE) ${ }^{3}$. El gobierno consideró que el argumento para prohibir el lucro en las escuelas no aplica para prohibirlo en las ATEs (Nicolás Eyzaguirre, Historia de la Ley N. ${ }^{\circ} 20.845$, p.319).

Con respecto al fin a la selección escolar, ésta quedo - tal como lo proponía el proyecto de leyestablecida solamente para las escuelas subvencionadas por el Estado, aunque en el primer trámite en la cámara de diputados, el ex líder del movimiento estudiantil Jackson ${ }^{4}$ presenta una indicación al artículo 12 que modifica al de la Ley General de Enseñanza para que las escuelas privadas no puedan seleccionar hasta sexto de primaria. Esta indicación, era acorde a la nueva idea de la educación como derecho social, sin embargo, la oposición reaccionó intentando frenar esta medida y contó con el apoyo de la Democracia Cristiana quienes solicitan la eliminación de esta indicación por considerar que no forma parte de las ideas matices de la ley legislar sobre las escuelas particulares (Ignacio Walker, Historia de la Ley $N^{\circ} 20.845$, p.1098). Además, desde la misma Democracia Cristiana se solicita que las escuelas subvencionadas por el Estado de alta exigencia académica o de especialización temprana puedan seleccionar a un $30 \%$ de sus vacantes desde primero de secundaria, lo que finalmente vendrá incluido en el artículo 7 de la ley.

Sobre el fin al financiamiento compartido, el Estado de forma progresiva asume el cobro que las escuelas particulares subvencionadas realizaban obligatoriamente a las familias, pero se establece que los padres podrán seguir aportando voluntariamente. Esto en el proyecto de ley se consideraba como una donación, por lo que el monto se descontaría del total de la subvención. Para revertir esto, un grupo de diputados principalmente del partido demócrata cristiano, presentan una indicación para crear un aporte voluntario que no tenga el mismo tratamiento de una donación. Así, con la aprobación de esta indicación, nace un nuevo concepto de aporte voluntario de los padres que no imputará a la subvención siempre y cuando no sean regulares y se utilicen exclusivamente para financiar actividades extracurriculares. Una vez aprobada la indicación, no se considera el fundamento de la educación como derecho social para el fin al financiamiento compartido que sostiene que los aportes económicos sobre la subvención estatal se traducen en una desigual distribución de la educación (Atria, 2014). Solamente se esboza cierta preocupación por considerar que el origen del aporte voluntario y del financiamiento compartido son similares (Yasna Provoste, Historia de la Ley No 20.845, p.1742).

\footnotetext{
${ }^{3}$ Las ATE surgen en Chile propiciadas por la ley de subvención escolar preferencial (SEP) en el año 2008, la cual permite que los dueños o administradores de las escuelas contraten con los recursos de la ley a estas agencias para mejorar sus resultados educativos. (Espínola y Silva, 2009).

${ }_{4}$ Presidente de la Federación de estudiantes de la Universidad Católica de Chile durante las movilizaciones del año 2011 y diputado de la República de Chile desde el año 2014.
} 


\section{DISCUSIÓN Y CONCLUSIONES}

La ley de inclusión escolar supone un avance, ya que por primera vez desde la vuelta a la democracia, el Estado establece condiciones a las escuelas particulares subvencionadas para mantener la denominada «igualdad de trato» (Falabella, 2015) entre estas escuelas y las públicas. Precisamente, el hecho de que el Estado durante aproximadamente dos décadas no haya establecido una distinción entre los ámbitos público y privado, contribuyó en que él mismo ha quedado disminuido frente a la gran relevancia que alcanzó el sector privado en educación lo que ha dificultado la transformación de las políticas públicas (Bellei, 2016). Esta problemática queda manifiesta en la oposición que encontró el gobierno en el parlamento para conseguir la aprobación de la ley, en donde debió consensuar no sólo con la derecha, sino que también dentro de su propia coalición para llevarla adelante. En efecto, estos consensos son los que impiden hablar de una reforma que efectivamente elimina al mercado de la educación chilena. Así por ejemplo, los servicios educativos externos pueden seguir lucrando, se permite - aunque con condiciones - que los padres sigan aportando a la educación de sus hijos y la selección de estudiantes queda permitida en ciertas escuelas. Además no se cambia la formulación tradicional del principio educativo chileno nacido en la reforma neoliberal de la educación, el cual reconoce a la familia como el principal actor educativo y por ende le da libertad para escoger la escuela de sus preferencias, así como tampoco se modifica el sistema de financiamiento a la demanda. Ambas prácticas, de acuerdo a Ball \& Youdell (2008) corresponden a la lógica de mercado en educación.

Finalmente, con la aprobación de la ley de inclusión escolar se busca eliminar tres prácticas de mercado de las escuelas financiadas con recursos públicos que los movimientos estudiantiles pusieron en cuestión. Con esta prohibición el sistema educativo chileno, si bien se acerca a prácticas habituales en países desarrollados de Norte América y Europa (ver Bellei, C., y Trivelli, C., 2015), no obstante transita solamente hacia un sistema educativo de cuasi-mercado (Bellei, 2016). Es por esto que resulta imposible afirmar que en Chile se ha reemplazado el paradigma de mercado en educación por el de derecho social tal como lo afirmaba la reforma.

\section{REFERENCIAS BIBLIOGRÁFICAS}

Atria, F., Larraín, G., Benavente, J.M., Couso, J., Joignant, A., (2013). El otro modelo: Del orden neoliberal al régimen de lo público, Santiago: Debate.

Atria, F. (2014). Derechos sociales y educación: un nuevo paradigma de lo público, Santiago: LOM.

Bachelet, M. (2013). Programa de Gobierno Michelle Bachelet 2014-2018. Recuperado de http://www.subdere.gov.cl/sites/ default/files/programamb_1.pdf.

Bachelet, M. (2015). Discurso de S.E. la Presidenta de la República, Michelle Bachelet, al promulgar Ley N. ${ }^{\circ} 20.845$ de Inclusión Escolar. Recuperado de https://2014-2018- prensa.presidencia.cl/discurso.aspx?id=16180.

Ball, S. J., \& Youdell, D. (2008). Hidden Privatization in Public Education. Education International, 1-66.

Bellei, C. (2016). Dificultades y resistencias de una reforma para des-mercantilizar la educación. RASE: Revista de La Asociación de Sociología de La Educación, 9(2), 232-247. https://doi.org/10.7203/rase.9.2.8417.

Bellei, C., Cabalin, C., \& Orellana, V. (2014). The 2011 Chilean student movement against neoliberal educational policies. Studies in Higher Education, 39(3), 426-440. https://doi.org/10.1080/03075079.2014.896179.

Bellei, C. \& Trivelli, C. (2015). Apoyo público a escuelas privadas en las experiencias de Europa y Norteamérica. En Bellei, C., El gran experimento. Mercado y privatización de la educación chilena. Santiago: LOM.

Biblioteca del Congreso Nacional de Chile (2015). Historia de la Ley 20.845. Recuperada de http://s.bcn.cl/1uoji.

Boric, G. (2012). Los horizontes del movimiento estudiantil. Le Monde Diplomatique. Recuperado de https://www.lemondediplomatique.cl/Los-horizontes-del-movimiento.html.

Cabalin, C. (2012). Neoliberal Education and Student Movements in Chile: Inequalities and Malaise. Policy Futures in Education, 10(2), 219-228. https://doi.org/10.2304/pfie.2012.10.2.219.

Cabalin, C., \& Bellei, C. (2013). Chilean Student Movements: Sustained Struggle to Transform a Market-oriented Educational System. Current Issues in Comparative Education, 15(2), 108-123. 
Canales, M., Bellei, C., \& Orellana, V. (2016). ¿Por qué elegir una escuela privada subvencionada? Sectores medios emergentes y elección de escuela en un sistema de mercado. Estudios Pedagógicos (Valdivia), 42(3), 89-109. https:// doi.org/10.4067/S0718-07052016000400005.

Espínola, V., \& Silva, M. E. (2009). Competencias del sostenedor para una efectiva gestión del mejoramiento educativo en el nivel local: Una propuesta. En Foco Educación, (3), 1-33.

Falabella, A. (2015). El mercado escolar en Chile y el surgimiento de la Nueva Gestión Pública: El tejido de la política entre la dictadura neoliberal y los gobiernos de la centroizquierda (1979 a 2009), Educação \& Sociedade, 36(132), 699-722.

Friedman, M. (1955). The Role of Government in Education. Economics and the Public Interest, ed. Robert A. Solo, by the Trustees of Rutgers College in New Jersey. Reprinted by permission of Rutgers University Press.

García-Huidobro, J. (2011). Movilizaciones estudiantiles, un intento de interpretación, (2008), 1- 10.Recuperado de http:// mailing.uahurtado.cl/cuaderno_educacion_35/pdf/actualidad35.pdf.

Garreton, M. A., Cruz, M. A., Aguirre, F., Bro, N., Farías, E., Ferreti, P., \& Ramos, T. (2011). Movimiento social , nuevas formas de hacer política y enclaves autoritarios. Polis, 10(30), 117-140.

Hsieh, C.-T., \& Urquiola, M. (2006). The effects of generalized school choice on achievement and stratification: Evidence from Chile's voucher program. Journal of Public Economics, 90(8-9), 1477-1503. https://doi.org/10.1016/J.JPUBECO.2005.11.002.

Jessop, B. (2010). Cultural political economy and critical policy studies. Critical Policy Studies, 3(3-4), 336-356. https://doi. org/10.1080/19460171003619741.

Jofré, G. (1988). El sistema de subvenciones en educación. La experiencia chilena. Estudios Públicos, 32, $192-237$.

Luengo, J.; Olmedo, A.; Santa Cruz, E. y Saura, G. (2012). Nuevas formas, nuevos actores y nuevas dinámicas de la privatización en la educación. Profesorado. Revista de currículum y formación del profesorado 16(3), 3-10.

Marshall, T. H. (1950). Citizenship and Social Class and Other Essays. Cambridge: Cambridge University Press.

Ministerio de Educación (2015). Cuenta pública año 2014.

Moulian, T. (1997) Chile anatomía de un mito. Santiago: LOM.

OECD (2013). Education at a Glance 2013: OECD Indicators, OECD Publishing. http://dx.doi.org/10.1787/eag-2013-en.

Parcerisa, L., \& Fallabella, A. (2017). La Consolidación del Estado Evaluador a Través de Políticas de Rendición de Cuentas: Trayectoria, Producción y Tensiones en el Sistema Educativo Chileno. Education Policy Analysis Archives, 25(89), 1-27. https://doi.org/10.14507/epaa.25.3177.

Picazo, M., \& Pierre, C. (2016). La educación como derecho social: La construcción del referencial de acción pública del movimiento estudiantil chileno, 25, 99-120.

Rodríguez, M. A. (2005). La transformación del Estado chileno: El caso de la Reforma Educacional de los 90. Berlin: Forschungs- und Dokumentationszentrum Chile- Lateinamerika e.V.

Ruiz, C. (2010). De la república al mercado. Santiago: LOM Ediciones.

Ruiz, C. (2012). La República, el Estado y el mercado en educación. Revista de Filosofía, 68, 11-28. https://doi.org/10.4067/ S0718-43602012000100003.

Schriewer, J. (1993). El método comparativo y la necesidad de externalización: criterios metodológicos y conceptos sociológicos. En Schriewer, J. y Pedró, F. (Eds.). Manual de educación comparada (Vol. II) Teorías, investigaciones, perspectivas. pp. 189-251. Barcelona: Pomares-Corredor.

Segovia, C., \& Gamboa, R. (2012). Chile: el año en que salimos a la calle. Revista de Ciencia Política, 32(1), 65-86. https:// doi.org/10.4067/S0718-090X2012000100004.

Valenzuela, J. P., Villalobos, C., \& Gomez, G. (2013). Segregación y polarización en el sistema escolar chileno y recientes tendencias: ¿Qué ha sucedido con los grupos medios? Espacio Público.

Vergara, J. (2012). El debate entre la educación republicana y la neoliberal en Chile. Polisemia, 8(13), 62. https://doi. org/10.26620/uniminuto.polisemia.8.13.2012.62-74.

Verger, A. (2016). The Global Diffusion of Education Privatization. In The Handbook of Global Education Policy (pp. 64-80). Chichester, UK: John Wiley \& Sons, Ltd. https://doi.org/10.1002/9781118468005.ch3.

Verger, A., Zancajo, A., \& Fontdevila, C. (2016). La economía política de la privatización educativa: políticas, tendencias y trayectorias desde una perspectiva comparada. Revista Colombiana de Educación, 70, 47-78. https://doi.org/10.17227/01203916.70rce47.78. 